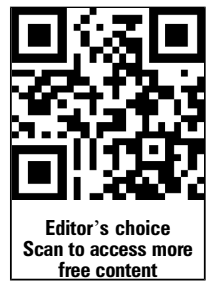

- Additional material is published online only. To view please visit the journal online (http://dx.doi.org/10.1136/ bjsports-2013-092646).

${ }^{1}$ Centre for Translational Neuroscience and Mental Health, School of Medicine and Public Health, University of Newcastle, Callaghan, New South Wales, Australia ${ }^{2}$ Department of Physical Medicine and Rehabilitation, Harvard Medical School; \& Red Sox Foundation and Massachusetts General Hospital Home Base Program, Boston, Massachusetts, USA

${ }^{3}$ The Florey Institute of Neuroscience and Mental Health, Melbourne Brain Centre-Austin Campus, Heidelberg, Victoria, Australia

\section{Correspondence to} Dr Andrew Gardner, Centre for Translational Neuroscience and Mental Health, Level 5, McAuley Building, Calvary Mater Hospital, Waratah, NSW 2298 , Australia, andrew.gardner@neurogard. com.au.

Accepted 30 May 2013 Published Online First 26 June 2013
To cite: Gardner A, Iverson GL, McCrory P. Br J Sports Med 2014;48:84-90.

\title{
Chronic traumatic encephalopathy in sport: a systematic review
}

\author{
Andrew Gardner, ${ }^{1}$ Grant L Iverson, ${ }^{2}$ Paul McCrory ${ }^{3}$
}

\section{ABSTRACT \\ Objective To provide a critical review of chronic} traumatic encephalopathy (CTE) by considering the range of clinical presentations, neuropathology and the strength of evidence for CTE as a distinct syndrome.

Data sources Seven electronic databases were searched using a combination of MeSH terms and key words to identify relevant articles.

Review methods Specific inclusion and exclusion criteria were used to select studies for review. Data extracted where present included study population, exposure/outcome measures, clinical data, neurological examination findings, cognitive assessment, investigation results and neuropathology results.

Results The data from 158 published case studies were reviewed. Critical differences between the older descriptions of CTE (the 'classic' syndrome) and the recent descriptions (the 'modern' syndrome) exist in the age of onset, natural history, clinical features, pathological findings and diagnostic criteria, which suggests that modern CTE is a different syndrome. The methodology of the current studies does not allow determination of aetiology or risk factors.

Conclusions The clinicopathological differences between the 'classic' CTE syndrome and the 'modern' syndrome suggest that the new syndrome needs a different nomenclature. Further research is required to clearly define the clinical phenotype of the modern CTE syndrome and establish the underlying aetiology. Future research needs to address these issues through largescale, prospective clinicopathological studies.

\section{INTRODUCTION}

Tremendous media attention surrounding sport-related concussion has been directed towards the potential for long-term problems in athletes with high exposure to head contact (ie, both concussive and subconcussive impacts) during a career in contact sport. ${ }^{1-7}$ This attention has been fuelled by the publication of autopsy case studies of retired professional athletes $^{8-11}$ and research reporting increased mortality rate due to neurodegenerative diseases in former professional athletes. ${ }^{12}$ There is also evidence from retrospective surveys ${ }^{8-11}$ supporting an association between long-term cognitive, psychiatric $^{13}$ and neurobehavioural problems and participation in sport.

In recent years, chronic traumatic encephalopathy (CTE) has been redefined from the original condition resembling Alzheimer's disease (AD) in professional boxers to a new condition observed in athletes, military personnel and civilians that shares many features with known psychiatric disorders and other forms of dementia. At present, the lack of operational criteria to confirm either a clinical or pathological diagnosis of CTE presents a considerable limitation to understanding this condition. ${ }^{14}$

A number of recent reviews have been published examining a diverse range of aspects associated with CTE. ${ }^{14-19}$ The purpose of this paper was to systematically review the evidence for CTE in sportsmen and sportswomen and to consider the important differences between the original description of CTE and the modern description of CTE.

\section{METHODS}

Articles were retrieved via online database searching, hand-searching reference lists and cited reference searches. Articles were limited to those printed in English language journals between 1850 and April 2013. The online databases searched were MEDLINE, CINAHL, EMBASE, Mosby's Index, PsycEXTRA, PsycINFO and Scopus. Key words, MeSH terms and combinations of these were used. Key words included CTE, dementia pugilistica, punch drunk syndrome, traumatic encephalopathy, CTE, repetitive head injury, sports concussion, multiple concussions, chronic concussions, subconcussive blow and sports-related traumatic brain injury. This search yielded 158 published case studies for review. Detailed information relating to demographic characteristics, athletic participation, concussion history, age of onset, clinical features, cognitive findings and neuropathological features is provided in online supplementary tables S1 and S2. The search methodology and hits are described in figure 1 using the PRISMA style. ${ }^{20}$

\section{'CLASSIC' CTE IN PROFESSIONAL BOXERS}

In 1928, Dr Harrison Martland, a medical examiner, presented 23 unverified cases believed to be experiencing a 'punch-drunk' state that had been described to him by a boxing promoter. He personally examined five but only described the clinical details of one in the article: a 38-year-old man who fought professionally for 7 years, having started his career at the age of 16 . The boxer had sustained two knockouts (one for a duration of 1 hour). Clinical examination, 20 years after the onset of the patient's symptoms, revealed that the boxer had tremor, gait ataxia and pyramidal tract dysfunction, but possessed normal intelligence. Martland's diagnosis was 'paralysis agitans' (Parkinson's disease (PD)) in this case. ${ }^{21}$

The incidence of classic CTE has proven difficult to establish, mostly due to a lack of high-quality prospective studies. Roberts ${ }^{22}$ studied 250 retired boxers from a cohort of 16781 UK boxers, registered between 1929 and 1955, and reported that lesions of the nervous system were present in 37 cases (17\% of total). It is important to note that 


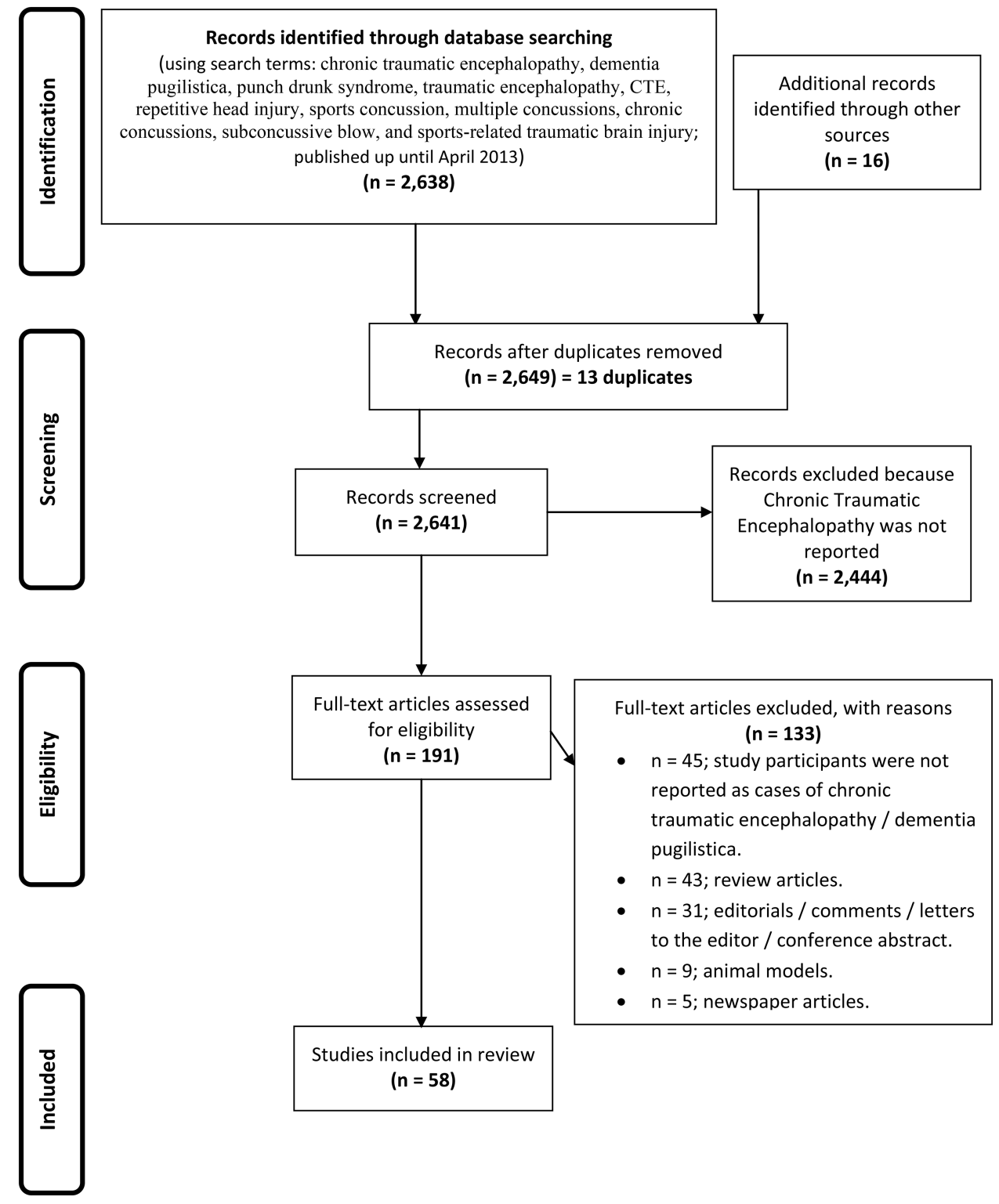

Figure 1 PRISMA flow diagram.

the oldest boxers in this cohort fought in the late 1800s, in an era where bare-knuckle championships were still conducted and there was little medical supervision or weight matching of boxers. Of the 37 cases reported by Roberts, the author provided details on only 11 (see online supplementary table S1). A careful review of the clinical details in professional boxers led McCrory et $\mathrm{al}^{23}$ to question the certainty of the 'CTE' diagnosis in most cases. In part, this lack of certainty reflects the relatively crude investigational technology available in the 1960s (eg, pneumoencephalograms as the only available brain imaging modality) at the time Roberts studied these retired boxers. The rates cannot be generalised to accurately reflect the prevalence levels in modern-day boxing because the current day boxers experience reduced exposure to repetitive head trauma and increased medical monitoring preparticipation. ${ }^{24}$

After reviewing the published cases, McCrory ${ }^{25}$ noted that cognitive deterioration was typically detected 10-20 years postretirement. Interestingly, the early descriptions noted that the physical signs and problems, but not the cognitive deficits, progressed postretirement. These retired athletes seemed to have one of two clinical syndromes. The first, present in about $70 \%$ of the cases, was characterised by dysarthria, pyramidal dysfunction and cognitive deficits and was not progressive. The second clinical syndrome (which was progressive) was characterised by dysarthria and pyramidal dysfunction, but with intact cognitive abilities (approximately 30\% of cases). 222627

An obvious challenge in attributing all the clinical features of these syndromes solely to a career in boxing is that some retired athletes have comorbidities that can contribute to neuropsychiatric problems, such as alcohol dependence, drug abuse, apolipoprotein E genotype ${ }^{28}$ and various medical problems. As such, clinical and autopsy cases need to be examined carefully. ${ }^{29}$

The neuropathology of classic CTE has been described in detail and includes (1) cavum septum pellucidum with septal fenestration; (2) cerebellar scarring involving Purkinje cell loss and thinning of the granular layer; (3) degeneration of the substantia nigra and locus coeruleus and (4) diffuse neurofibrillary tangles (NFTs) involving the medial temporal region, 
uncus, amygdala, hippocampus, parahippocampal gyrus and fusiform gyrus along with the more lateral temporal, insular and frontal cortices. ${ }^{30}$

The neuropathological burden appears to correlate with the level of boxing exposure as measured by the duration of career and the number of bouts ${ }^{30}$ (see online supplementary table S2). Roberts et $a l^{27}$ re-examined 14/15 brains originally described by Corsellis et $a l,{ }^{30}$ as well as six additional boxers' brains using immunocytochemistry, and 19/20 cases also demonstrated widespread diffuse $\beta$-amyloid deposits and $\tau$. Schmidt et al ${ }^{31}$ found that the $\tau$ positive NFTs observed in two boxers with pathological evidence of CTE were identical in both isoform ratio and phosphorylation state to those observed in $\mathrm{AD}$. These two cases continue to be the only examples of classic CTE where detailed biochemical analysis has been conducted. ${ }^{14}$

Classic CTE does not appear to advance in a predictable and sequential series of stages, ${ }^{32} 33$ and progression of physical symptoms is only present in approximately one-third of cases. ${ }^{22}$ The extent to which age-related changes, psychiatric or mental-health illness, alcohol/drug use or coexisting dementing illnesses contribute to this process is largely unaccounted for in the published literature. The case descriptions contain extensive histories of psychiatric illnesses, severe substance abuse and other medical or neurological problems that might contribute to or confound the neuropathological findings. In addition, the potential genetic risk in those former athletes with a family history of neurodegenerative disease and the extent to which this contributes to the clinical and pathological profiles also require further investigation. ${ }^{23}$

\section{'MODERN' CTE}

Four independent research groups have reported case studies of retired athletes believed to have CTE. ${ }^{11}{ }^{34-36}$ The original publications ${ }^{37} 38$ introduced changes in the clinical features and the neuropathology as compared with the classic entity described by Roberts et $a l^{27}$ and Corsellis et $a l^{30}$ in their boxing patients. One research group defined CTE as the "chronic cognitive and neuropsychiatric symptoms of chronic neurodegeneration following a single episode of severe traumatic brain injury or (more commonly) repeated episodes of mild traumatic brain injury." 39 This subtle definitional shift makes it unclear as to whether we are now dealing with a new condition (modern CTE vs classic CTE), a variant of a single disease or another unrelated pathology (eg, frontotemporal dementia (FTD)).

The 'modern' form of CTE, since 2005, encompasses a broad spectrum of clinical symptoms and pathological findings in former athletes. $^{24} 40$ When compared with the classical CTE entity, there are differences in exposure rates, clinical presentation, cognitive and neurobehavioural features, diagnostic criteria, age of symptom onset, disease progression and putative neuropathological characteristics (see table 1). The incidence of modern CTE is unknown; it has been estimated at less than 4\% of professional American football players, based on the numbers of cases obtained in a given period versus the number of athletes who died during the same period. If all of these professional athletes at risk were to be used as the exposure, then the incidence rate would be less than $0.01 \%$.

The initial case of modern CTE was reported by Omalu et al in 2005, with two additional cases reported in 2006 and 2010, and further cases reported in 2011 (for review, see Refs. 9, 35, 37 and 45). Of the 17 cases reported by Omalu et $a l^{35}, 11$ (approximately 65\%) were identified as having neuropathology consistent with a diagnosis of CTE. Hazrati et $a l^{34}$ examined the brains of six former Canadian Football League players who presented with neurological decline and three $(50 \%)$ demonstrated neuropathological changes consistent with CTE; however, these cases also had vascular disease, $\mathrm{AD}$ and cancer comorbidities. The other three cases were diagnosed with $\mathrm{AD}$, ALS and Parkinson's disease. ${ }^{34}$ McKee et al ${ }^{11}$ also reported on two retired boxers. ${ }^{8}$ In their recent large neuropathological case series involving 85 patients, consisting of athletes $(n=64$, including 33 former NFL players) and military veterans $(n=21)$, $80 \%$ showed at least some evidence of the neuropathology reported to characterise CTE (see Discussion section below).

\section{Signs and symptoms}

Although the modern CTE description describes gait disorders, speech slowing and extrapyramidal signs, neuropsychiatric and behavioural symptoms reportedly predominate early and are prominent throughout the course of the condition. ${ }^{22} 4647$ These neuropsychiatric symptoms include mood disorder (mainly depression), paranoia, agitation, social withdrawal, poor judgement and aggression. Cognitive impairment tends to emerge later ${ }^{33} 46$ and typically includes impairment across the domains of orientation, memory, language, attention, information processing speed and executive functioning. ${ }^{47}$ Less characteristic of classic CTE, cognitive impairment in modern CTE reportedly progresses over time. ${ }^{8} 11$ Disease staging and disease phenotypes of modern CTE (table 2) have now been proposed based on the level of neuropathological evidence of disease on autopsy rather than clinical presentation. ${ }^{1135}$

It may be that the description of the modern CTE symptomatology is confounded by the retrospective nature of the data collection and the heavily biased case selection. Consequently, the reported clinical observations (ie, suicidality, emotional lability, aggression and disinhibition) are likely to be skewed by selection bias. $^{14}$

In a recent review article, Jordan ${ }^{17}$ proposed clinical criteria for the diagnosis of CTE which classified clinical features into four categories in line with other neurological diseases: definite, probable, possible and improbable CTE. He recommended that, in light of the lack of currently available biomarkers to observe the natural history of CTE, characterisation of preclinical and prodromal CTE (similar to the preclinical phases that have been documented in $\mathrm{AD}$ ) is premature. ${ }^{17}$

\section{IS THERE A PRECLINICAL CTE STATE?}

Although no longitudinal studies have been performed describing the clinical phenotype or the progression of neuropathology, there is evidence from retrospective surveys supporting an association between participation in elite sport and long-term cognitive, psychiatric and neurobehavioural problems. ${ }^{8-11} 4849$ In many cases, the causes for these outcomes are multifactorial and numerous methodological confounds exist. However, there is some neurophysiological and radiological evidence suggesting that persistent or prolonged disturbance of the brain function may occur following concussive injury. ${ }^{44}$ 50-54 Preliminary data in a small positron emission tomography imaging study suggest that subcortical and amygdala $\tau$ deposition was detected in vivo in five retired American football players who exhibited mood and cognitive symptoms. ${ }^{55}$ It is possible that a subgroup of athletes exhibit persistent clinical or physiological dysfunction with associated neuropathology.

\section{Neuropathology}

Of the 61 pure athlete (with only a background in sport) cases reported by McKee et al, ${ }^{11} 15$ (or 25\%) demonstrated no pathological features of CTE (cases: 19-32, 34), 4 (approximately 7\%) also fulfilled criteria for an $\mathrm{AD}$ diagnosis (cases: 87, 
Table 1 Features of classic versus modern CTE

\begin{tabular}{|c|c|c|}
\hline Characteristic & Classic CTE & Modern CTE \\
\hline $\begin{array}{l}\text { Number of reported } \\
\text { cases }\end{array}$ & $\begin{array}{l}\text { Approximately } 250(11 \text { detailed })^{27} \\
\text { Approximately } 15^{30} \\
\text { Approximately } 10^{41} \\
\text { Approximately } 6^{42} \\
\text { Approximately } 3^{43}\end{array}$ & $\begin{array}{l}85 \text { (17 not considered CTE pathology })^{8} 11 \\
17(6 \text { not considered CTE pathology })^{35}\end{array}$ \\
\hline Age of onset & Late 50 s-early $60 \mathrm{~s}^{22}$ & $\begin{array}{l}\text { Staging of the disease }{ }^{11} \\
\text { Stage I: } 22.2 \text { years ( } \mathrm{SD}=5.6 \text { years) } \\
\text { Stage II: } 39.0 \text { years ( } 9.3 \text { years) } \\
\text { Stage III: } 44.3 \text { years ( } 10.7 \text { years) } \\
\text { Stage IV: } 57.2 \text { years ( } 15.4 \text { years) } \\
\text { Early } 40 \mathrm{~s}^{35}\end{array}$ \\
\hline $\begin{array}{l}\text { Age at death } \\
\text { range (mean) }\end{array}$ & $57-91$ years $^{30}$ & $\begin{array}{l}14-98 \text { years (mean: } 54.1 \text { years } \pm 23.3 \text { years) }{ }^{11} \\
18-52 \text { years (mean: } 41 \text { years) }\end{array}$ \\
\hline Exposure & $\begin{array}{l}60-700+\text { bouts and/or } 7-25 \text { years' boxing }{ }^{27} \\
>50 \% \text { of sample had } 300+\text { bouts and/or } 15 \text { years' boxing. A number of } \\
\text { cases also fought in an unknown number of booth contests }{ }^{30}\end{array}$ & $\begin{array}{l}5 \text { and } 24 \text { years as a professional boxer; unreported number of bouts } \& 48 \\
\text { professional bouts, respectively }{ }^{8} \text { otherwise unreported career lengths/ } \\
\text { exposure; primarily American football, hockey, and military exposure } \\
\text { No data on the career lengths/exposure; level of play-high school, } \\
\text { collegiate and NFL football; professional boxing; wrestling; MMA }{ }^{35}\end{array}$ \\
\hline Progression & $30 \%$ progress - physical symptoms may progress & $\begin{array}{l}\text { Yes, mean } 18.6 \text { years }{ }^{8} \text {; Yes; noted in first three cases, but no progression } \\
\text { reported in other cases }\end{array}$ \\
\hline Clinical features & $\begin{array}{l}\text { Dysarthria, movement difficulties (slowing and 'unsteady on feet'), } \\
\text { tremor, later onset of memory problems, alcohol sensitivity }\end{array}$ & $\begin{array}{l}\text { Neuropsychiatric and behavioural symptoms prominent. Cognitive deficits } \\
\text { and dementia with progression }\end{array}$ \\
\hline Genotype risk & ApoE $4^{28}$ & No ApoE 4 genotype risk ${ }^{11}$ \\
\hline Diagnosis & Clinically: clinical assessment and limited radiological evaluation ${ }^{27} 30$ & Neuropathologically confirmed on autopsy \\
\hline $\begin{array}{l}\text { Classic } \\
\text { neuropathology }\end{array}$ & \multicolumn{2}{|c|}{$\begin{array}{l}\text { Septal fenestration, cerebellar scarring (inferior surface of the lateral lobes), degeneration of the substantia nigra and locus coeruleus, diffuse } \\
\text { neurofibrillary tangle inclusion (medial temporal region, uncus, amygdala, hippocampus, parahippocampal gyrus, fusiform gyrus, lateral temporal, } \\
\text { insular and frontal cortices). NFTs spread diffusely through the cerebral cortex and brainstem, with vast concentrations in the medial temporal grey, } \\
\text { uncus, corticomedial part of the amygdala nucleus, hippocampus, parahippocampal nucleus, fusiform gyrus, lateral temporal area, insula and frontal } \\
\text { cortex, with an absence of senile plaques }{ }^{30}\end{array}$} \\
\hline $\begin{array}{l}\text { Modern } \\
\text { neuropathology }\end{array}$ & \multicolumn{2}{|c|}{ 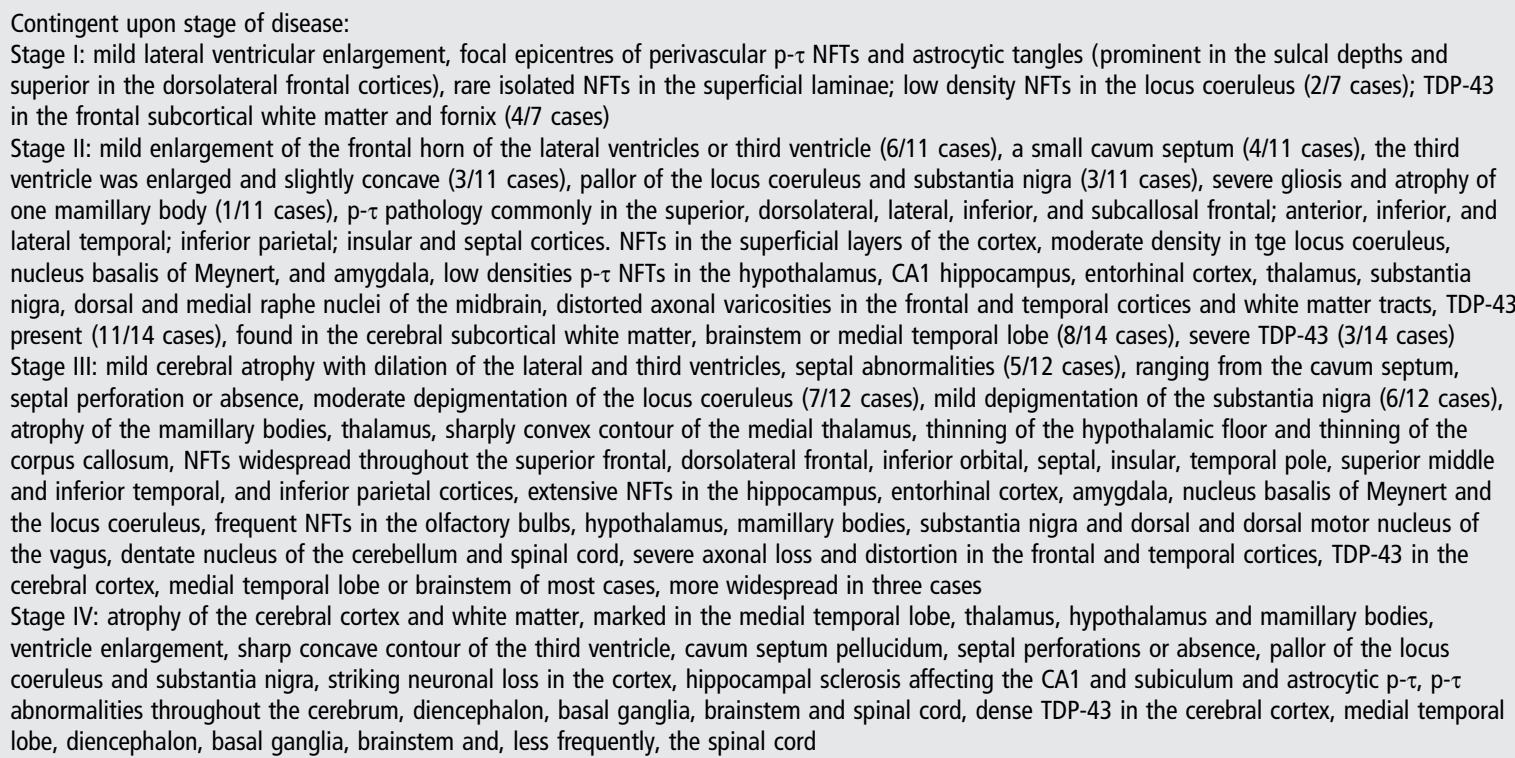 } \\
\hline
\end{tabular}

CTE, chronic traumatic encephalopathy; NFTs, neurofibrillary tangles; $p-\tau$, phosphorylated $\tau$ protein.

89, 91, 92), 4 (approximately 7\%) possessed Lewy bodies (cases: 92, 95, 96, 97), 3 (or approximately 5\%) demonstrated FTD pathology (cases: 100, 102, 103) and 3 (approximately 5\%) showed evidence of PD (cases: 91, 100, 101). Of these 'pure athlete' cases, only 15 (approximately 25\%; cases: 36, 39, $41,43,45,50,52,58,60,63,64,67,68,72,77)$ had 'pure CTE pathology' (defined as having any clinical symptoms and pure pathology (with TPD-43+ as a minimum), and the absence of any other neurodegenerative disease and/or no other non-CTE neuropathology). However, within these 15 'pure athlete, pure CTE' cases, there was not always a consistent association between pathology and clinical presentation (eg, cases 43 and 68 were initially considered to be asymptomatic and only subsequently reported to have neurobehavioural features; and case 36 's only symptom was a headache).

When both athlete and military cases were considered, CTE was the sole diagnosis in 43 cases (or approximately 70\%), with a greater proportion of these 'pure CTE' cases represented in stages III and IV disease, where a more florid pathology has been reported. ${ }^{11}$ The pathophysiological hallmark of these cases 
Table 2 Histopathological classification of modern CTE

BU CSTE criteria-four stages of disease ${ }^{11}$
Stage 1
Normal brain weight. Focal epicentres of perivascular $p-\tau$ and NFTs and astrocytic tangles involving the
sulcal depths and typically affecting the superior and dorsolateral frontal cortices

Stage 2

Normal brain weight. Multiple epicentres at the depths of the sulci with localised spread from epicentres to the superficial layers of the adjacent cortex. No NFTs or $p-\tau$ involvement in the medial temporal lobe

\section{Stage 3}

Mild reduction in brain weight. Mild cerebral atrophy with dilation of the lateral and third ventricles. Septal abnormalities. Moderate depigmentation of the locus coeruleus and mild depigmentation of the substantia nigra. Atrophy of the mamillary bodies and thalamus. Widespread $p-\tau$ pathology in the frontal, insula, temporal and parietal lobes. NF pathology in the amygdala, hippocampus and entorhinal cortex

\section{Stage 4}

Marked reduction in brain weight with atrophy of the cerebral cortex. Marked atrophy of the medial temporal lobe, thalamus, hypothalamus and mamillary bodies. Severe $p-\tau$ pathology affecting most regions of the cerebral cortex and the medial temporal lobe, sparing the calcarine cortex. Severe $p-\tau$ pathology in the diencephalon, basal ganglia, brainstem and spinal cord. Marked axonal loss of subcortical WM tracts

\section{Omalu et al-four phenotypes ${ }^{35}$}

Phenotype I

Sparse to frequent NFTs and neuritic threads in the cerebral cortex and brainstem but without involvement of the subcortical nuclei (basal ganglia) and cerebellum. No diffuse amyloid plaques in the cerebral cortex

Phenotype II

Sparse to frequent NFTs and neuritic threads in the cerebral cortex and brainstem with or without such pathology in the subcortical nuclei (basal ganglia) and cerebellum. Diffuse amyloid plaques in the cerebral cortex

Phenotype III

Brainstem predominant: moderate to frequent NFTs and neuritic threads in the brainstem nuclei, absent or sparse NFTs and neuritic threads in the cerebral cortex, subcortical nuclei (basal ganglia) and cerebellum. No amyloid plaques in the cerebral cortex

Phenotype IV

Absent or sparse NFTs and neuritic threads in the cerebral cortex, brainstem, and subcortical nuclei (basal ganglia). No cerebellar involvement. No diffuse amyloid plaques in the cerebral cortex

CTE, chronic traumatic encephalopathy; LC, locus coeruleus; NF, neurofibrillary; NFTs, neurofibrillary tangles; $\mathrm{p}-\tau$, phosphorylated $\tau$; SNr, substantia nigra; WM, white matter.

is the presence of regionally specific $\tau$-immunoreactive NFTs, astrocytic tangles and neuropil neurites observed in both the cortical and subcortical regions. ${ }^{8} 11$

The reported neuropathological characteristics of both entities (classic and modern CTE) share a number of common features such as fenestrated septum pellucidum, cerebral atrophy, NFT inclusion (although found in greater amounts in modern CTE), $\beta$ amyloid deposition (found in greater amounts in classic CTE), reduced pigmentation of the substantia nigra and locus coeruleus and enlarged ventricles. Modern CTE in the latter stages also includes frontotemporal lobe atrophy with extensive $\tau$ pathology and TDP43 + distributed throughout the neocortex, medial temporal lobe, diencephalon, brainstem and spinal cord (see online supplementary table S2). A comparison of TDP-43 pathology with the classic CTE cases is not possible because detailed TDP-43 studies have not been performed on the classic CTE cases. Gavett et $a l^{56}$ reviewed 51 cases that included both classic and modern CTE patients and noted that diffuse amyloid plaques were found in $47 \%$, neuritic amyloid plaques in $27 \%$ and amyloid angiopathy in $6 \%$. Similar findings were noted by McKee et al. ${ }^{11}$

Although many similarities in neuropathological findings have been reported by the two primary research groups studying CTE, there are also some differences. McKee et $a l^{8}{ }^{11}$ reported a marked accumulation of $\tau$-immunoreactive astrocytes as a hallmark feature, but this was not observed in the cases examined by Omalu et $a l .^{35}$ Omalu et $a l^{35}$ reported a lobar cortical distribution but not a prominent periventricular topographic distribution, whereas McKee et $a l^{8}$ noted that the initial pathological changes were perivascular and at the base of the sulci and in the later stages were primarily affecting the frontotemporal regions. Both groups have attempted to provide a pathological classification of modern CTE. McKee and colleagues have classified CTE into four stages, while Omalu and colleagues have described four phenotypes of CTE (see table 2).

\section{BRAIN PATHOLOGY IN NORMAL AGEING}

Longitudinal aging studies provide unique and important information relating to clinical features, neuropathology and the relation between clinical features and neuropathology. For example, during the Honolulu-Asia Aging Study, ${ }^{57}$ follow-up clinical and neuropsychological evaluations have been conducted with 3508 men who were free of dementia at the study onset and a total of 593 brain autopsies have been completed to date (for review see Ref. 58).

Postmortem brain examinations demonstrate diverse pathology. For example, in older adults clinically diagnosed as 'pure AD', fewer than $50 \%$ of these people had the typical pathological features of AD. Moreover, neuropathological abnormalities are found in approximately $40 \%$ of neuropsychologically normal patients. ${ }^{58-60}$ Similar differences between the clinical presentation (ie, living diagnosis) and postmortem neuropathology have also been reported in other aging study samples. ${ }^{57} 6162$ For example, the Nun Study is a longitudinal study of aging and AD in 678 Catholic sisters. The participants were aged 75-102 years at the beginning of the study in $1991 .^{63}{ }^{64}$ Interestingly, results from this study have revealed that a substantial proportion of the nuns who demonstrated mild (58\%) and moderate (32\%) stages of AD neuropathology did not show evidence of memory impairment. Even in the nuns with the most severe expression of $\mathrm{AD}$ neuropathology, a small percentage $(8 \%)$ did not demonstrate memory impairment. ${ }^{63}$ Further, the Framingham Heart Study, which has recruited three generations of families in a longitudinal study, reported $75 \%$ sensitivity (27/36 patients with pathologically verified $\mathrm{AD}$ were clinically diagnosed with $\mathrm{AD}$ ) and $92.9 \%$ specificity (52/56 patients without AD were correctly identified as not having $\mathrm{AD}) .^{65}$

In a recent study of former NFL players, ${ }^{12}$ the mortality rate from neurodegenerative disease was three times greater than that of the general population. However, the sample size in this study was small with only two cases of AD and six ALS cases in the total sample of 334 cases. Although this is an intriguing observation, the study lacked power to be definitive.

It is clear from these aging studies that neuropathological abnormalities are not necessarily correlated with clinical features and may be seen in cognitively normal older adults. Therefore, assumptions regarding causal relationships between neuropathology and clinical presentation should proceed 
cautiously, with careful consideration for methodological limitations, moderating variables and possible alternative explanations (see online supplementary table S2 in the article by Nelson et $a l^{66}$ ). Clinicopathological correlations will be more reliable after using matched control observations and adequately sized series. ${ }^{14}$

\section{DISCUSSION}

We identified 158 autopsy cases in the literature that have been examined for CTE. There are important differences in the modern version of CTE, described in recent autopsy cases, and the classic description of 'dementia pugilistica' that create confusion in the understanding of these conditions. There are critical differences in age of onset, progression, clinical features, pathological findings and diagnostic criteria that suggest we might be dealing with two or more conditions. Of the 85 autopsies that have been performed in athletes over the past 10 years, $20 \%$ had 'pure' neuropathology consistent with CTE, 52\% had CTE plus other neuropathology, 5\% had neuropathology but no CTE and 24\% had no neuropathology (see table 3).

Although the clinical phenotype is not yet clarified, CTE is characterised by distinct neuropathological findings. ${ }^{8} \quad 1135 \quad 56$ The distribution of $\tau$-immunoreactive astrocytes distinguishes the modern CTE entity, with preferential involvement of the superficial cortical layers occurring on a background of relative scarcity of $\beta$-amyloid plaques, which is more characteristic of the classical CTE entity. The unique characteristic of modern CTE appears to be the location of the neuropathology, in the grey matter and perivascular space at the depth of sulci, moreso than the specific proteins or lesion types. These findings however, need to be independently verified.

In light of the currently available evidence, a conservative operational definition for the modern CTE disease process is provided below.

1. A presenting clinical profile that includes (but is not limited to) cognitive (eg, attention and memory) and mental health problems.

2. Neuropathology that includes only the neurofibrillary phosphorylated- $\tau$ and TDP-43+ observed in the characteristically described regional pattern, in the absence of other neuropathology such as amyloid and $\alpha$-synuclein.

3 . The absence of any other disease or disorder that may conceivably explain the clinical or pathological findings.

In elderly patients, it is important to appreciate that neuropathological comorbidity is very common, and this can influence the clinical features. In $\mathrm{AD}$, more than two-thirds of patients demonstrate neuropathological comorbidities including cerebrovascular diseases, synucleinopathies, tauopathies, frontotemporal lobar degeneration and TDP-43-related diseases

Table 3 Modern CTE neuropathology in athletes

\begin{tabular}{lrrrrrrr}
\hline & McKee & Omalu & Hazrati & NIH & Total & Per cent \\
\hline Number of autopsy cases & 61 & 17 & 6 & 1 & 85 & - \\
Pure CTE neuropathology & 15 & 2 & 0 & 0 & 17 & 20.0 \\
CTE + other neuropathology & 31 & 9 & 3 & 1 & 44 & 51.8 \\
Neuropathology but no CTE & 0 & 1 & 3 & 0 & 4 & 4.7 \\
No neuropathology & 15 & 5 & 0 & 0 & 20 & 23.5 \\
\hline
\end{tabular}

CTE, chronic traumatic encephalopathy; $\mathrm{CTE}^{+}$, CTE and other neuropathology; NIH, National Institutes of Health. (see Ref. 66, p. 373). These findings have the potential to skew correlations between neuropathological findings and clinical or cognitive features. In the recent publication of CTE cases, $80 \%$ demonstrated CTE; of those cases $63 \%$ were pathologically diagnosed with 'pure CTE' and the remaining 37\% possessed pathological comorbidity. ${ }^{11}$

At present, a diagnosis of CTE can only be made postmortem. The spectrum of neuropathology in cognitively intact older adults and older adults with neurological problems is often diverse, not unitary, which adds methodological complexity to clincopathological correlational studies. In the various case series presented to date, $20-50 \%$ of those with clinical features did not show the neuropathology of CTE, and 5\% of those with the neuropathology did not show clinical features.

Furthermore, the clinical phenotype of modern CTE is unclear. The reported clinical features are tremendously broad, ranging from an isolated symptom of headache to severe neuropsychiatric illness and dementia. The clinical features overlap with a number of known psychiatric conditions and neurodegenerative diseases. The recent characterisation of $\mathrm{CTE}^{39}$ does not adequately consider plausible differential diagnoses, mediating variables, moderating variables or multifactorial neuropathological processes.

At present, there are no published epidemiological, crosssectional or prospective studies relating to CTE. The classical entity $^{22}$ was estimated to occur in approximately $17 \%$ of the exposed populations (this may be an overestimate given the diagnostic uncertainty) and it has been suggested that modern CTE occurs in fewer than 5\% of professional American football players ${ }^{67}$-although the actual prevalence is unknown.

The strongly presented causal assumptions in the literature relating to concussive and subconcussive brain impact exposure derived from the case studies are scientifically premature, especially given the absence of cross-sectional, epidemiological, prospective or longitudinal studies on the topic. The recently published case series ${ }^{11}$ adds considerably to the knowledge base relating to the neuropathology of CTE. In view of the relatively limited number of cases examined, together with the potential case-selection bias in autopsy-based studies, in addition to the limitations associated with verifying clinicopathological correlation, further research is required in order to better delineate this putative disease process.

It is important to conduct systematic research in the area to address specific unanswered questions. First, it is not known whether similar, or even identical, neuropathological findings are observed in other samples that share clinical characteristics with CTE such as patients with drug or steroid abuse, alcohol abuse histories, chronic psychiatric problems, cardiovascular/ cerebrovascular disease or other health conditions. Second, the extent to which the reported underlying neuropathology contributes to the reported clinical features (eg, cognitive deficits, psychiatric features) is uncertain. Third, the potential existence of a genetic contribution to the observed neuropathology has not been determined. Fourth, possible mediator or moderator variables for the association between the neuropathology and the clinical features have not been identified. Finally, we do not have a methodology for identifying individuals who are at future risk or who might currently have CTE. Psychiatric problems and cognitive impairment usually have multifactorial, not unitary causation-this will require further attention in future studies. The important next step in the process of potentially answering some of the unresolved issues associated with CTE is to conduct large-scale, prospective, longitudinal, clinicopathological studies. 


\section{What are the new findings?}

- The definition of chronic traumatic encephalopathy (CTE) has changed from the original 'classic' description seen in boxers.

- The clinical phenotype of CTE is unknown and cannot be diagnosed in vivo.

- A large scale prospective, longitudinal clinicopathological study is required to answer some of the currently unresolved issues associated with CTE.

Acknowledgements The Florey Institute of Neuroscience and Mental Health acknowledges the strong support from the Victorian Government and in particular the funding from the Operational Infrastructure Support Grant.

Contributors All authors were involved in the design, analysis and writing of the manuscript and each author had the right of veto over the publication.

Competing interests AG has a clinical practice in neuropsychology involving individuals who have sustained sports-related concussion (including current and former athletes). He has received travel funding from the Australian Football League (AFL) to present at a Concussion in Football Conference in 2013. Previous grant funding includes the NSW Sporting Injuries Committee, the Brain Foundation and the Hunter Medical Research Institute, supported by Jennie Thomas. Gl, PhD has been reimbursed by the government, professional scientific bodies and commercial organisations for discussing or presenting research relating to mild TBI and sport-related concussion at meetings, scientific conferences, and symposiums. He has a clinical and consulting practice in forensic neuropsychology involving individuals who have sustained mild TBIs (including professional athletes). He has received research funding from several test publishing companies, including ImPACT Applications, Inc, CNS Vital Signs and Psychological Assessment Resources (PAR, Inc). He is a coinvestigator, collaborator or consultant on grants relating to mild TBI funded by several organisations, including, but not limited to, the Canadian Institute of Health Research, Alcohol Beverage Medical Research Council, Rehabilitation Research and Development (RR\&D) Service of the US Department of Veterans Affairs, Vancouver Coastal Health Research Institute and Roche Diagnostics Canada. PMC currently receives financial research support from the National Health and Medical Research Council, the University of Melbourne, Victorian Department of Planning and Community Development, Sport and Recreation Division and the Eastern Health Network. Previous competitive grant funding includes the Australian Research Council, International Rugby Board, the University of Melbourne, the University of Otago (NZ), National Hockey League (USA), VicHealth, Australian Football League Research Foundation, Royal Australasian College of Surgeons and the Australian Sports Commission. He has a clinical and consulting practice in neurology and sports medicine involving individuals who have sustained concussion and TBI. He has received travel funding from the Medical Commission of the International Olympic Committee (IOC), the International Football Federation (FIFA), the American Academy of Neurology and the Jockey Club (UK). He receives book royalties from McGraw-Hill and from 2001-2008 was employed by the British Medical Journal Publishing Group. He has conducted clinical drug trials on anti migraine (Glaxo-Welcome; Janssen-Cliag; Novartis; Parke-Davis; Schering) and antispasticity drugs (Ipsen) through the Eastern Health Clinical Trials Unit in Melbourne. This drug trial work has not involved any financial payment to PMC directly. He received consultancy fees in 2010 from Axon Sports (USA) for the development of educational material (which was not renewed) and has received support since 2001 from CogState Inc for research costs and the development of educational material. He is a cofounder and shareholder in two biomedical companies (involved in eHealth and Compression garment technologies) but does not hold any individual shares in any company related to concussion or brain injury assessment or technology.

Provenance and peer review Not commissioned; internally peer reviewed.

\section{REFERENCES}

1 Schwarz A. Head injuries in football. New York Times 21 October 2010.

2 Gregory S. The problem with football: how to make it safer. TIME Magazine 2010:36-43.

3 Schwarz A. Expert ties ex-player's suicide to brain damage. New York Times 2007.

4 King P. The hits that are changing football. Sports Illustrated. 2010.

5 Jackson DZ. The black hole of sports. The Boston Globe. 2012.

6 Maki A. Fomer CFLer Doug Maclver had CTE. The Globe and Mail 17 September 2012.
7 Kaye AH, McCrory P. Does football cause brain damage? Available evidence suggests anecdotal media reports need to be assessed carefully. Med J Aust 2012;196:547-9

8 McKee AC, Cantu RC, Nowinski CJ, et al. Chronic traumatic encephalopathy in athletes: progressive tauopathy after repetitive head injury. J Neuropathol Exp Neurol 2009;68:709-35.

9 Omalu BI, Hamilton RL, Kamboh Ml, et al. Chronic Traumatic Encephalopathy (CTE) in a National Football League Player: case report and emerging medicolegal practice questions. J Forensic Nurs 2010;6:40-6.

10 Guskiewicz KM, Marshall SW, Bailes JE, et al. Association between recurrent concussion and late-life cognitive impairment in retired professional football players. Neurosurgery 2005;57:719-26.

11 McKee AC, Stein TD, Nowinski CJ, et al. The spectrum of disease in chronic traumatic encephalopathy. Brain 2013;136:43-64.

12 Lehman EJ, Hein MJ, Baron SL, et al. Neurodegenerative causes of death among retired National Football League players. Neurology 2012;79:1970-4.

13 Cantu RC. Chronic traumatic encephalopathy in the National Football League. Neurosurgery 2007;61:223-5.

14 Smith DH, Johnson VE, Stewart W. Chronic neuropathologies of single and repetitive TBI: substrates of dementia? Nat Rev Neurol 2013;9:211-21.

15 DeKosky ST, Blennow K, Ikonomovic MD, et al. Acute and chronic traumatic encephalopathies: pathogenesis and biomarkers. Nat Rev Neurol 2013;9:192-200.

16 Zetterberg $\mathrm{H}$, Smith DH, Blennow K. Biomarkers of mild traumatic brain injury in cerebrospinal fluid and blood. Nat Rev Neurol 2013;9:201-10.

17 Jordan BD. The clinical spectrum of sport-related traumatic brain injury. Nat Rev Neurol 2013;9:222-30.

18 Roozenbeek B, Maas AIR, Menon DK. Changing patterns in the epidemiology of traumatic brain injury. Nat Rev Neurol 2013;9:231-6.

19 McCrory P, Meeuwisse WH, Kutcher JS, et al. What is the evidence for chronic concussion-related changes in retired athletes: behavioural, pathological and clinical outcomes? BJSM 2013;47:327-30.

20 Liberati A, Altman DG, Tetzlaff J, et al. The PRISMA statement for reporting systematic reviews and meta-analyses of studies that evaluate health care interventions: explanation and elaboration. J Clin Epidemiol 2009;6:1-28.

21 Martland HS. Punch drunk. JAMA 1928;91:1103-7.

22 Roberts $\mathrm{AH}$. Brain damage in boxers: a study of the prevalence of traumatic encephalopathy among ex-professional boxers. London, UK: Pitman, 1969.

23 McCrory P, Zazryn T, Cameron P. The evidence for chronic traumatic encephalopathy in boxing. Sports Med 2007;37:467-76.

24 Clausen M, Anderson V, McCrory P. The risk of chronic traumatic brain injury in professional boxing: change in exposure variables in professional boxing over the past century. BJSM 2005;39:661-5.

25 McCrory P. Sports concussion and the risk of chronic neurological impairment. Clin J Sport Med 2011;21:6-12.

26 Johnson J. Organic psychosyndromes due to boxing. Br J Psychiatry 1969;115:45-53.

27 Roberts GW, Allsop D, Bruton C. The occult aftermath of boxing. J Neurol Neurosurg Psychiatry 1990;53:373-8.

28 Jordan $\mathrm{BD}$, Relkin NR, Ravdin LD, et al. Apolipoprotein E epsilon4 associated with chronic traumatic brain injury in boxing. JAMA 1997;278:136-40.

29 Nowak LA, Smith GG, Reyes PF. Dementia in a retired world boxing champion: case report and literature review. Clin Neuropathol 2009;28:275-80.

30 Corsellis JA, Bruton CJ, Freeman-Browne D. The aftermath of boxing. Psychol Med 1973:3:270-303

31 Schmidt ML, Zhukareva V, Newell KL, et al. Tau isoform profile and phosphorylation state in dementia pugilistica recapitulate Alzheimer's disease. Acta Neuropathol 2001;101:518-24.

32 La Cava G. Boxer's encephalopathy. J Sports Med Phys Fitness 1963;168:87-92.

33 Guterman A, Smith R. Neurological sequelae of boxing. Sports Med 1987:4:194-210

34 Hazrati LN, Tartaglia MC, Diamandis P, et al. Absence of chronic traumatic encephalopathy in retired football players with multiple concussions and neurological symptomatology. Front Hum Neurosci 2013;in press.

35 Omalu BI, Bailes J, Hamilton RL, et al. Emerging histomorphologic phenotypes of chronic traumatic encephalopathy in American athletes. Neurosurgery 2011;69:173-83.

36 Samson K. NIH: NFL'S Junior Seau had chronic traumatic encephalopathy. Neurol Today 2012;13:12-15.

37 Omalu BI, DeKosky ST, Minster RL, et al. Chronic traumatic encephalopathy in a National Football League Player. Neurosurgery 2005;57:128-34.

38 McKee AC, Cantu RC, Nowinski CJ, et al. Chronic traumatic encephalopathy in athletes: progressive tauopathy after repetitive head injury. J Neuropathol Exp Neurol 2009;68:709-35.

39 Omalu BI, Bailes J, Hammers JL, et al. Chronic traumatic encephalopathy, suicides and parasuicides in professional American athletes: the role of the forensic pathologist. Am J Forensic Med Pathol 2010;31:1-3.

40 DeKosky ST, Ikonomovic MD, Gandy S. Traumatic brain injury-football, warfare, and long-term effects. N Engl J Med 2010;363:1293-6. 
41 Maudsley C, Ferguson FR. Neurological disease in boxers. Lancet 1963;19:799-801.

42 Payne EE. Brains of boxers. Supplementum ad Fortschritte der Neurologie und Psychiatrie 1968:11:173-88.

43 Geddes JF, Vowles GH, Nicoll JA, et al. Neuronal cytoskeletal changes are an early consequence of repetitive head injury. Acta Neurol Belg 1999:98:171-8.

44 Vagnozzi R, Tavazzi B, Signoretti S, et al. Temporal window of metabloic brain vulnerability to concussions: mitochondrial-related impairment-part I. Neurosurgery 2007:61:379-89.

45 Omalu BI, DeKosky ST, Hamilton RL, et al. Chronic traumatic encephalopathy in a National Football League Player: part II. Neurosurgery 2006;59:1086-93.

46 Jordan BD. Chronic traumatic brain injury associated with boxing. Semin Neurol 2000:20:179-85

47 Costanza A, Weber K, Gandy S, et al. Review: Contact sport-related chronic traumatic encephalopathy in the elderly: clinical expression and structural substrates. Neuropathol Appl Neurobiol 2011;37:570-84.

48 Guskiewicz KM, Marshall SW, Bailes J, et al. Recurrent concussion and risk of depression in retired professional football players. Med Sci Sports Exerc 2007;39:903-9.

49 Schwenk TL, Gorenflo DW, Dopp RR, et al. Depression and pain in retired professional football players. Med Sci Sports Exerc 2007;39:599-605.

50 De Beaumont $\mathrm{L}$, Theoret $\mathrm{H}$, Mongeon $\mathrm{D}$, et al. Brain function decline in healthy retired athletes who sustained their last sports concussion in early adulthood. Brain 2009;132:695-708

51 Gardner A, Kay-Lambkin F, Stanwell P, et al. A systematic review of diffusion tensor imaging findings in sports-related concussion. J Neurotrauma 2012;29:2521-38.

52 Vagnozzi R, Signoretti S, Tavazzi B, et al. Temporal window of metabolic brain vulnerability to concussion: a pilot $1 \mathrm{H}$-magnetic resonance spectroscopic study in concussed athletes—part III. Neurosurgery 2008;62:1286-96.

53 Vagnozzi R, Signoretti S, Cristofori L, et al. Assessment of metabolic brain damage and recovery following mild traumatic brain injury: a multicentre, proton magnetic resonance spectroscopic study in concussed patients. Brain 2010;133:3232-42.

54 Vagnozzi R, Signoretti S, Floris R, et al. Decrease in N-acetylaspartate following concussion may be coupled to decrease in creatine. J Head Trauma Rehab 2012; [Epub ahead of print].
55 Small GW, Kepe V, Siddarth P, et al. PET scanning of brain tau in retired National Football League players: preliminary findings. Am J Geriatr Psychiatry 2013:21:138-44

56 Gavett BE, Stern RA, McKee AC. Chronic traumatic encephalopathy: a potential late effect of sport-related concussive and subconcussive head trauma. Clin Sports Med 2011;30:179-88.

57 Cruz KS Santa, Sonnen JA, Pezhouh M Kherad, et al. Alzheimer disease pathology in subject without dementia in 2 studies of aging: the Nun Study and the Adult Changes in Thought Study. J Neuropathol Exp Neurol 2011;70:832-40.

58 White LR. Brain lesions at autopsy in older Japanese-American men as related to cognitive impairment and dementia in the final years of life: a summary report from the Honolulu-Asia Aging Study. J Alzheimer Dis 2009;18:713-25.

59 Robinson R. Multiple pathologies in many Alzheimer disease patients. Neurol Today 2011;11:28-9.

60 Gelber RP, Launer LJ, White LR. The Honolulu-Asia Aging Study: epidemiologic and neuropathologic research on cognitive impairment. Curr Alzheimer Res 2012:9:664-72.

61 Snowden DA. Healthy aging and dementia: findings from the Nun Study. Ann Intern Med 2003:139(5 Pt 2):450-4.

62 Sonnen JA, Larson EB, Crane PK, et al. Pathological correlates of dementia in a longitudinal, population-based sample of aging. Ann Neurol 2007:62:406-13.

63 Snowden DA. Healthy aging and dementia: findings for the Nun Study. Ann Intern Med 2003:139:450-4

64 Snowden DA. Aging and Alzheimer's disease: lessons from the Nun Study. Gerontologist 1997;37:150-6.

65 Au R, Seshadri S, Knox K, et al. The Framingham Brain Donation Program: neuropathology along the cognitive continuum. Curr Alzheimer Res 2012:9:673-86.

66 Nelson PT, Alafuzoff I, Bigio EH, et al. Correlation of Alzheimer disease neuropathology changes with cognitive signs: a review of the literature. J Neuropathol Exp Neurol 2012;71:362-81.

67 Randolph C, Kirkwood MW. What are the real risks of sports-related concussion, and are they modifiable? J Int Neuropsycho/ Soc 2009;15:512-20. 